\title{
AVALIAÇÃO DA VIRULENCIA DE BLASTOSPOROS DE Metarhizium anisopliae NO CONTROLE DE LARVAS DE CAMPO DO MOSQUITO Aedes aegypti
}

\author{
Aline Teixeira Carolino ${ }^{1}$ \\ Simone Azevedo Gomes ${ }^{2}$ \\ Thalles Cardoso Mattoso ${ }^{3}$ \\ Thais Berçot Pontes Teodoro 4 \\ Richard Ian Samuels ${ }^{5}$
}

Resumo: Atualmente no Brasil, o mosquito Aedes aegypti é transmissor das arboviroses dengue, chikungunya e Zika. Não existe tratamento específico para estas doenças. A redução da população do vetor ainda é o método mais eficaz para reduzir a taxa dessas arboviroses. O presente estudo comparou a virulência de conídios e blastosporos de Metarhizium anisopliae contra larvas do mosquito A. aegypti provenientes de coletas no campo. Blastosporos foram mais virulentos para larvas, sendo observada mortalidade total das larvas em apenas 48 horas. Larvas infectadas com conídios apresentaram $100 \%$ de mortalidade no quinto dia pós-infecção. O presente estudo mostra que blastosporos apresentam grande potencial para controle de larvas de $A$. aegypti no campo.

Palavras-chave: Fungo entomopatogênico; Virulência; Dengue; Chikungunya; Zika.

${ }^{1}$ Ciências Biológicas / Universidade Estadual Norte Fluminense, Brasil. E-mail: carolinoat@gmail.com.br. ${ }^{2}$ Ciências Biológicas/ Universidade Estadual Norte Fluminense, Brasil. E-mail: simoneazgomes@yahoo.com.br. ${ }^{3}$ Agronomia / Universidade Estadual Norte Fluminense, Brasil. E-mail: thallesmattoso@hotmail.com. ${ }^{4}$ Ciências Biológicas / Universidade Estadual Norte Fluminense, Brasil. E-mail: thaisbercot@yahoo.com.br. ${ }^{5}$ Zoologia / Universidade Estadual Norte Fluminense, Brasil. E-mail: richardiansamuels@gmail.com. 